\title{
Energy-Loss Characteristics for EFTEM Imaging with a Liquid Flow Cell
}

\author{
K.L. Klein, ${ }^{*}$ N. de Jonge, ${ }^{* *}$ and I.M. Anderson* \\ *Surface and Microanalysis Science Division, National Institute of Standards and Technology, \\ Gaithersburg MD 20899-8371 \\ **Department of Molecular Physiology and Biophysics, Vanderbilt University Medical Center, \\ Nashville TN 37232-0615
}

The imaging of microscopic structures at nanometer-scale spatial resolution in a liquid environment is of interest for a wide range of studies, with applications from nanotherapeutics to homogeneous catalysis. We have recently demonstrated that bright-field imaging in the transmission electron microscope (TEM) using a prototype liquid flow cell specimen holder provides a promising method for meeting this challenge [1]. However, the imaging characteristics for TEM are rapidly degraded with increasing projected thickness of the liquid cell, as the incident electron beam undergoes multiple elastic and inelastic scattering. In particular, a small collection semi-angle $\beta$ and energyselecting slit $\Delta E$ are required to maintain nanometer-scale spatial resolution in the face of spherical and chromatic aberrations, respectively, which greatly limits the available signal. In this paper, we report on various challenges for performing energy-filtered TEM (EFTEM) imaging in a liquid cell.

EFTEM zero-loss (ZL), low-loss (LL) and spectral images (SI) were acquired with incident energy $E_{0}=300 \mathrm{keV}$ and a small effective beam convergence semi-angle $\alpha<1 \mathrm{mrad}$. In order to separate the contributions of the amorphous silicon nitride $\left(\mathrm{SiN}_{\mathrm{x}}\right)$ membranes from those of the encapsulated fluid, we began by measuring the variation of $t / \lambda$ with $\beta$, as shown in Fig. 1. Data acquired with two instruments, and with $t / \lambda$ maps formed both from the simple ratio of LL and ZL images and through extraction from a SI $(\triangle E=5 \mathrm{eV} ; 0 \leq E \leq 100 \mathrm{eV})$, indicated a significant and reproducible variation, indicating that $\lambda(\beta)$ varies by $\approx 30 \%$ as $\beta$ increases from $\beta=2.7 \mathrm{mrad}$ to $\beta=10.8 \mathrm{mrad}$. The higher image intensity that results from increasing the collection angle is thus rendered less useful by the increased proportion of inelastically scattered electrons.

The elastic deformation of the 50-nm-thick $\mathrm{SiN}_{\mathrm{x}}$ membrane under the pressure differential from the liquid-filled cell to the vacuum presents a more significant challenge for liquid-cell imaging. A plot of the calculated bulge height $h$ as a function of membrane radius $r$ and thickness $t$, shown in Fig. 2, indicates that reducing the lateral dimension of the electron-transparent window provides the most effective strategy for minimizing the thickness variation across the window. An electron energy-loss (EEL) spectrum profile illustrates the dramatic effect of the bulging membrane on the energy-loss spectrum for an electron-transparent window of width $90 \mu \mathrm{m}$, as shown in Fig. 3. The EEL spectral shape evident near the edge of the membrane (2b) degrades into a featureless distribution of intensity with a peak energy and energy breadth of several hundred $\mathrm{eV}$ at the membrane center. The correspondingly large variation in intensity and specimen thickness are evident in Fig. 4 [3].

[1] K.L. Klein, I.M. Anderson, and N. de Jonge, J. Microsc. (2011) available online Jan. 20, 2011. doi:10.1111/j.1365-2818.2010.03484.x

[2] F.S. Tsai, Characterization of Mechanical and Optical Properties of X-ray Mask Membranes, RLE Technical Report No.564, Massachusetts Institute of Technology, Cambridge MA (1991).

[3] We are grateful to Protochips Inc. for access to their prototype TEM holder and for support from the National Research Council Postdoctoral Research Associateship Program (KLK). 

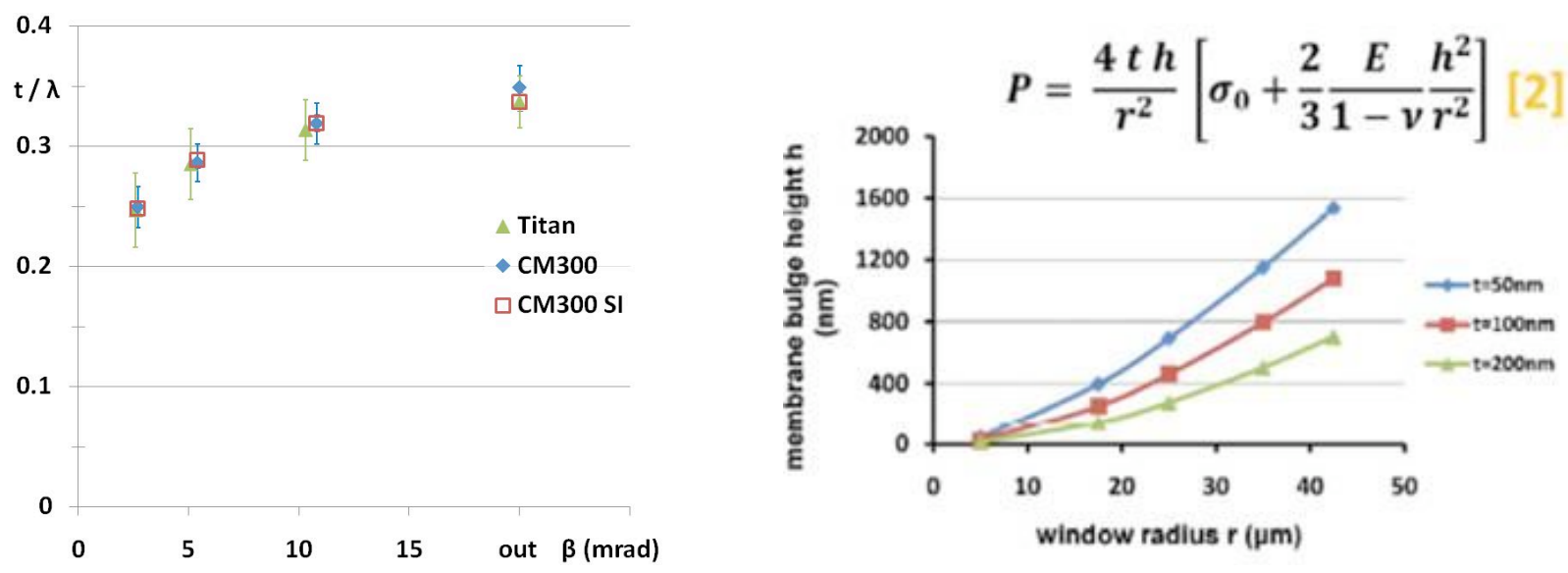

FIG. 1. Plot of measured $t / \lambda$ values for 50 -nm-thick amorphous $\operatorname{SiN}_{\mathrm{x}}$ as a function of collection aperture semi-angle $\beta$. Since thickness $t$ is constant, clearly $\lambda=\lambda(\beta)$.

FIG. 2. Pressure differential $(P)$ dependence and calculated bulge height $h$ as a function of radius $r$ of electron-transparent $\operatorname{SiN}_{\mathrm{x}}$ window of thickness $t$. Here, $\sigma_{0}$ is the residual $(P=0)$ stress, $E$ is Young's modulus, and $v$ is Poisson's ratio for the $\mathrm{SiN}_{\mathrm{x}}$ membrane [2].

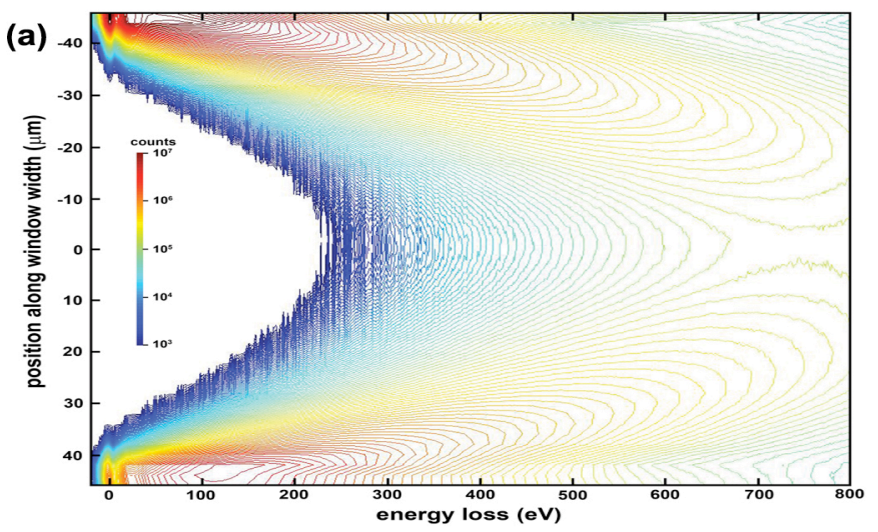

(b)

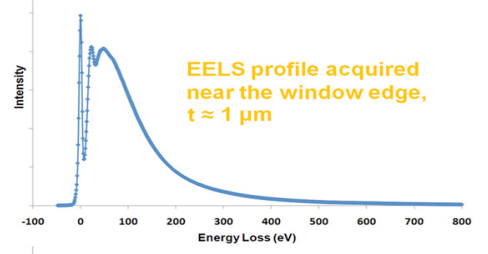

(c)

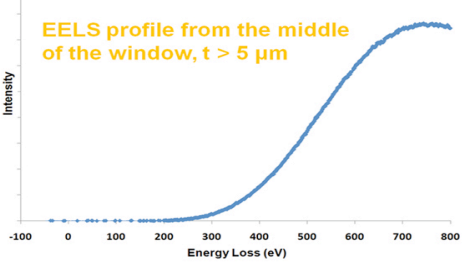

FIG. 3. Effect of membrane bulging on EEL characteristics of liquid cell: (a) spectrum profile contour plot, showing intensity as a function of energy loss and position relative to the edge of a 90 $\mu \mathrm{m}$-wide $\operatorname{SiN}_{\mathrm{x}}$ window; $(\mathrm{b}, \mathrm{c})$ EEL spectra from (b) edge $(-45 \mu \mathrm{m})$ and (c) center $(0 \mu \mathrm{m})$ of window.
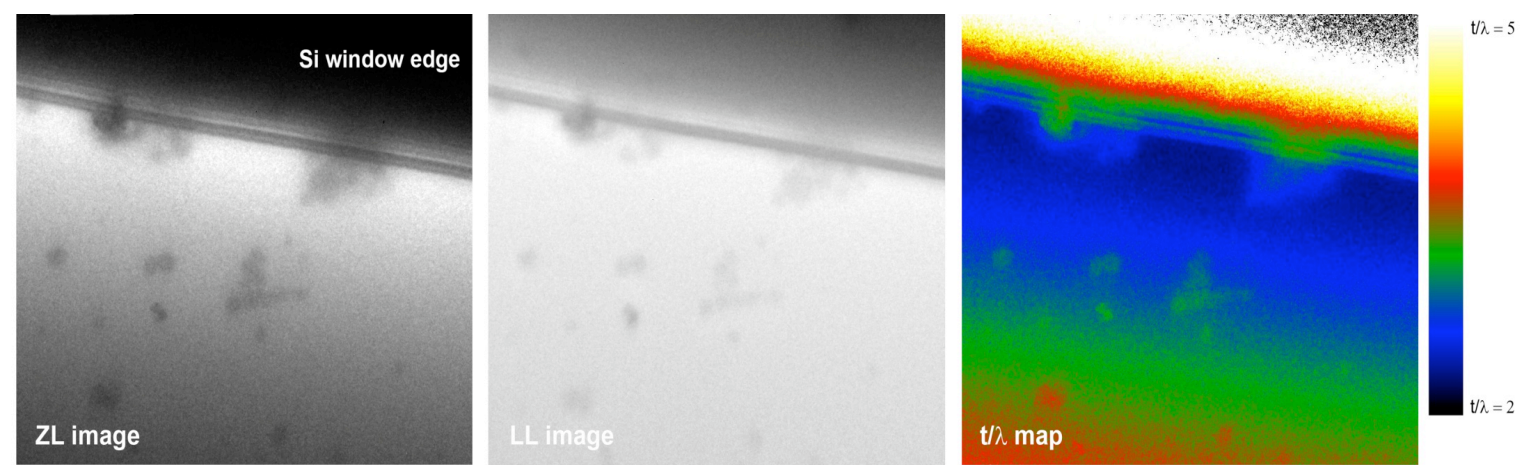

FIG. 4. Zero loss (ZL), low loss (LL) and $t / \lambda$ images of citrate-stabilized gold nanoparticles suspended in the liquid cell of Fig. 2. For scale, images are $2.5 \mu \mathrm{m}$ in height. The bulging increases the thickness of the cell by a factor of 2 within $2 \mu \mathrm{m}$ of the window's edge, from $t / \lambda \approx 2$ to $t / \lambda \approx 4$. 